\title{
Shot-noise-limited laser power stabilization with a high-power photodiode array
}

\author{
Patrick Kwee, ${ }^{1,2, *}$ Benno Willke, ${ }^{1,2}$ and Karsten Danzmann ${ }^{1,2}$ \\ ${ }^{1}$ Max-Planck-Institut für Gravitationsphysik (Albert-Einstein-Institut), 30167 Hannover, Germany \\ ${ }^{2}$ Leibniz Universität Hannover, 30167 Hannover, Germany \\ *Corresponding author: patrick.kwee@aei.mpg.de
}

Received June 19, 2009; revised August 8, 2009; accepted August 13, 2009;

posted August 25, 2009 (Doc. ID 113046); published September 18, 2009

\begin{abstract}
The output power of a cw Nd:YAG laser was stabilized in a dc-coupled feedback loop with a low-noise multiphotodiode detector and an electro-optic amplitude modulator in the frequency band from $1 \mathrm{~Hz}$ to $1 \mathrm{kHz}$. For the first time, to our knowledge, an independently measured relative power noise of $2.4 \times 10^{-9} \mathrm{~Hz}^{-1 / 2}$ at $10 \mathrm{~Hz}$ was achieved, fulfilling the power stability requirements of the Advanced LIGO (Laser Interferometer Gravitational-Wave Observatory) gravitational wave detector. () 2009 Optical Society of America

OCIS codes: $140.3425,040.5160$.
\end{abstract}

For many optical precision experiments it is necessary to stabilize the laser power, since power fluctuations can often limit the sensitivity of these experiments. Today some of the most demanding power stability requirements are defined by laser interferometers for the detection of gravitational waves (GWs) [1-3]. Power fluctuations in the GW frequency band cause radiation pressure fluctuation that shakes the suspended test mass, which can mask possible GW signals. Furthermore, next-generation GW detectors will use the dc-readout technique [4], where laser power fluctuations directly couple to the GW signal. For the second-generation detector Advanced Laser Interferometer Gravitational-Wave Observatory (LIGO) [5], a maximum relative power noise of only $2 \times 10^{-9} \mathrm{~Hz}^{-1 / 2}$ at $10 \mathrm{~Hz}$ is tolerable. To meet this requirement an active power stabilization of the laser system is necessary.

In this Letter we report a power stabilization of a cw solid-state laser at $1064 \mathrm{~nm}$ wavelength with a low-noise multiphotodiode detector in a dc-coupled feedback loop. We used an electro-optic amplitude modulator (EOAM) as the actuator to stabilize the power in a frequency band from $1 \mathrm{~Hz}$ to $1 \mathrm{kHz}$. As recognized in previous stabilization experiments [6-8], the control loop sensor is the most critical part, and it is quite demanding to reach a shot-noiselimited sensitivity for a photocurrent of $100 \mathrm{~mA}$ and more, especially at frequencies as low as $10 \mathrm{~Hz}$. With a power detector consisting of an array of four photodiodes in a pressure-tight tank, we detected a total photocurrent of about $200 \mathrm{~mA}$. Even though multiple photodiodes were already used for heterodyne signal detection at radio frequencies $[9,10]$, this is to our knowledge the first low-noise dc-coupled application of photodiode arrays in a power stabilization experiment.

We achieved an independently [out-of-loop (OOL)] measured relative power noise of $2.4 \times 10^{-9} \mathrm{~Hz}^{-1 / 2}$ at $10 \mathrm{~Hz}$ and $1.8 \times 10^{-9} \mathrm{~Hz}^{-1 / 2}$ for frequencies above 15 $\mathrm{Hz}$, thus fulfilling the Advanced LIGO requirements. Our measurement was limited by electronic noise and shot noise. To our knowledge this is the first ex- periment in this power stability range for which the limiting noise sources were fully identified, which was closer than $1 \mathrm{~dB}$ to the shot-noise limit for frequencies above $15 \mathrm{~Hz}$ and which fulfilled the Advanced LIGO requirements.

In our experimental setup (Fig. 1) we used a Nd:YAG nonplanar ring oscillator (NPRO), (Innolight Mephisto $2000 \mathrm{NE}$ ) as the laser. An EOAM (Thorlabs EO-AM-NR-C2) along with a subsequent polarizing beam splitter was used as the laser power actuator. A ring resonator and the photodiode array were placed in a pressure-tight tank for acoustic shielding. The tank was pumped down and vented with high efficiency particulate air (HEPA) filtered air to reduce the number of particles potentially passing the laser beam. The ring resonator with a design similar to the one in [11] was used as the MC and reduced beam pointing fluctuations. The finesse was about 370 with

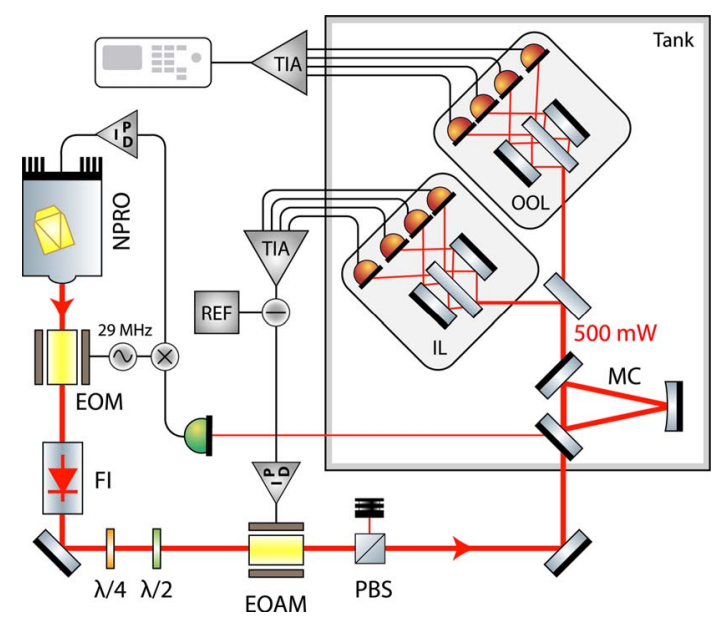

Fig. 1. (Color online) Experimental setup. The laser (NPRO) was locked to the mode cleaner (MC) inside a pressure-tight tank. Power fluctuations were measured with four in-loop (IL) photodiodes to stabilize the laser power using an EOAM. The OOL detector was used to independently measure the power stability achieved. EOM, electro-optic phase modulator; FI, Faraday isolator; PBS, polarizing beam splitter; TIA, transimpedance amplifier; REF, voltage reference; $\lambda / 2, \lambda / 4$, wave plates. 
a bandwidth of about $980 \mathrm{kHz}$. The beam size on the photodiodes was changed with a lens, and a mirror glued to a three-axis piezoelectric element was used to create pointing modulations downstream of the MC (this lens and this mirror are omitted in Fig. 1 for clarity).

The beam $(\approx 500 \mathrm{~mW})$ was split with $50: 50$ beam splitters into eight partial beams of nearly equal power (57-65 $\mathrm{mW}$ ) using multiple reflections. These beams hit the eight photodiodes at an incidence angle of $45^{\circ}$ in horizontal polarization. The glass windows of the photodiode packages were removed to avoid spurious reflections, and the beams reflected at the photodiode surfaces were absorbed in Schott BG39 glass filters at the Brewster angle. By this means, back reflections and scattering of the detectors were minimized. The beam radii on the photodiodes were about $545 \pm 14$ and $386 \pm 10 \mu \mathrm{m}$ in the horizontal and the vertical directions, respectively.

The photodiode array consisted of eight Perkin Elmer C30642 InGaAs photodiodes with an active diameter of $2 \mathrm{~mm}$. Each photodiode could be moved transversely by $\pm 1 \mathrm{~mm}$ to its incident beam to align the photodiode. The photodiodes were aligned to the position of the smallest coupling to lateral beam jitter, which was determined by modulating the beam pointing. Each photodiode was mounted electrically isolated to an aluminum plate, which was also used as a heat sink.

The electronics (Fig. 2) for the photodiodes were placed outside the tank. Each photodiode was connected with a pair of shielded cables $(\approx 70 \mathrm{~cm})$ to a filtered $5 \mathrm{~V}$ bias voltage and a low-noise transimpedance amplifier with a low current-noise $200 \Omega$ resistor. (We observed no performance difference with cables extended to a length of $5 \mathrm{~m}$.) At the operation point each photodiode detected a photocurrent of about $50 \mathrm{~mA}$. The average responsivity of the photodiodes was $0.79 \pm 0.08 \mathrm{~A} / \mathrm{W}$.

The eight signals of the transimpedance amplifiers were amplified between $3 \mathrm{~Hz}$ and $2.5 \mathrm{kHz}$. Four signals were added and used to stabilize the laser power IL, and the remaining four signals were added and used to verify the power stability (OOL). With pluggable tank feedthroughs the assignment of the photodiodes to the IL and OOL readout electronics was easily changeable. For the stabilization the IL signal was subtracted from a low-pass filtered voltage reference, amplified in analog servo electronics, and fed back to the EOAM. This dc-coupled feedback control loop had a bandwidth of about $80 \mathrm{kHz}$ with a loop gain of more than $68 \mathrm{~dB}$ for frequencies below $1 \mathrm{kHz}$. The OOL signal was passed through an antialiasing filter and was differentially transmitted to an analogto-digital converter card in a computer. The time se-

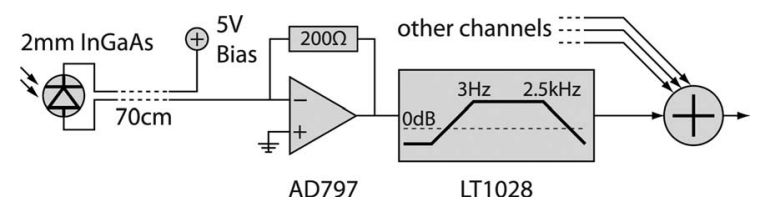

Fig. 2. Simplified schematic of the photodiode readout electronics. ries of the signal was recorded and used to calculate the linear spectral density of the relative power noise between $1 \mathrm{~Hz}$ and $1 \mathrm{kHz}$.

The readout and the servo electronics were each connected to an independent power supply. We found no performance difference with battery packs as power supplies.

To measure the combined electronic noises of the transimpedance amplifier, the signal conditioning, the signal adder, and the digitization at the operation point, we replaced the photodiode with a low-noise current source (800 $\Omega$ output impedance) built of batteries. It should be noted that the electronic noise measured in this way was higher than without any current flowing through the transimpedance amplifier (dark noise).

We found a free-running relative power noise downstream of the $\mathrm{MC}$ of $1 \times 10^{-7}-1 \times 10^{-6} \mathrm{~Hz}^{-1 / 2}$ in the frequency band from $1 \mathrm{~Hz}$ to $1 \mathrm{kHz}$. With closed feedback loop, the OOL measured power noise (Fig. 3 ) was at the expected level defined by the uncorrelated sum of the shot and the electronic noises of both the IL and OOL detectors in the whole frequency band. For frequencies up to $7 \mathrm{~Hz}$, the measured noise was dominated by the electronic noise of the IL and OOL detectors and for higher frequencies by shot noise at a level of $1.8 \times 10^{-9} \mathrm{~Hz}^{-1 / 2}$. The relative shot noise level of this experiment $N_{\mathrm{S}}=\left(2 e / I_{\mathrm{il}}+2 e / I_{\mathrm{ool}}\right)^{1 / 2}$ $=1.8 \times 10^{-9} \mathrm{~Hz}^{-1 / 2}$ was calculated using the total IL $\left(I_{\mathrm{il}}=200 \mathrm{~mA}\right)$ and OOL $\left(I_{\text {ool }}=189 \mathrm{~mA}\right)$ photocurrents. The measured power noise was closer than $1 \mathrm{~dB}$ (our estimated calibration accuracy was $1 \mathrm{~dB}$ ) to the shotnoise level for frequencies from $15 \mathrm{~Hz}$. At $10 \mathrm{~Hz}$ we measured a relative power noise of $2.4 \times 10^{-9} \mathrm{~Hz}^{-1 / 2}$ on the OOL detector.

Since the IL and OOL detectors were built equally, we assumed that the noise of the IL and the OOL detectors contributed equally to the measurement noise. With this assumption and by subtracting the noise contribution of the OOL detector, we deduced a $3 \mathrm{~dB}$ lower relative power noise of the laser beam of $1.7 \times 10^{-9} \mathrm{~Hz}^{-1 / 2}$ at $10 \mathrm{~Hz}$ and $1.3 \times 10^{-9} \mathrm{~Hz}^{-1 / 2}$ at

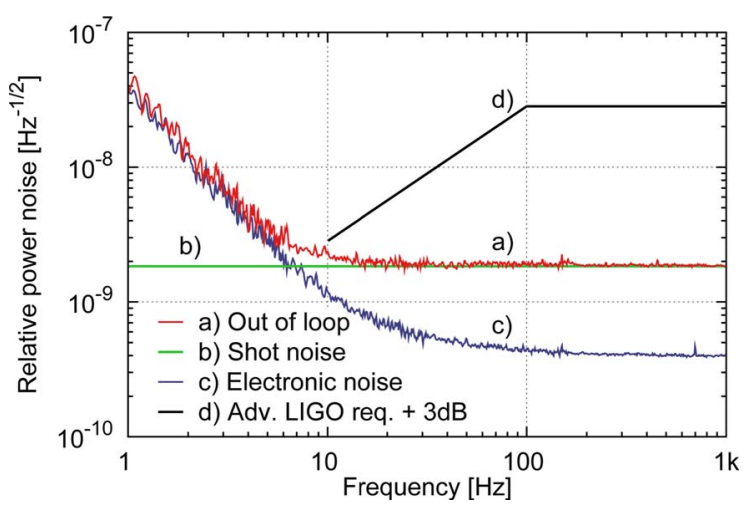

Fig. 3. (Color online) OOL measured power noise. For low frequencies the measurement was limited by electronic noise and for frequencies above $7 \mathrm{~Hz}$ by shot noise of the IL (200 mA photocurrent) and OOL (189 mA photocurrent) detectors. The Advanced LIGO power noise requirement, shifted by $3 \mathrm{~dB}$ in the plot to account for the noise of the OOL detector, was met in the whole frequency band. 
higher frequencies and thereby demonstrated the Advanced LIGO power noise requirements [Fig. 3(d)].

The experiment showed the described performance for periods of up to $6 \mathrm{~h}$, only interrupted by relocks of the MC owing to thermal drifts. By careful cabling, independent power supplies, and differential signal transmission, all peaks at the power line frequency of $50 \mathrm{~Hz}$ and harmonics were eliminated in the measurement.

A coupling of lateral beam jitter to the OOL relative power noise of $2.8 \pm 0.3$ and $1.0 \pm 0.1 \mathrm{~m}^{-1}$ in the horizontal and vertical directions was measured using the mirror attached to the piezoelectric element. The deduced upper bounds for beam jitter at the photodiodes were 0.7 and $2 \mathrm{~nm} \mathrm{~Hz}^{-1 / 2}$ in the horizontal and vertical directions, respectively. Before we aligned each photodiode to minimize the beam jitter coupling, we aligned them to the responsivity maximum and got a coupling of about $50 \mathrm{~m}^{-1}$-also many peaks at mechanical resonance frequencies were visible in the power noise.

We observed no performance difference of the power stabilization between the situations when the tank was filled with the HEPA filtered air and when it was evacuated $(\approx 100 \mathrm{~Pa})$. With unfiltered laboratory air (particle count of about $8500 \mathrm{~m}^{-3} \approx 250 \mathrm{ft}^{-3}$, particle size of $\geq 0.3 \mu \mathrm{m}$ ), it was impossible to take a stationary measurement owing to signal glitches caused by particles.

We also found no performance difference depending on which photodiodes were used for the IL and the OOL signals. This indicated that the splitting ratio of the beam splitters was stable enough for our measurement.

Finally, in a separate experiment we measured the power-stability dependence on the beam size at the photodiodes. We used the same readout electronics, but only two photodiodes to speed up the alignment process. We observed no performance difference for beam radii from 60 to $720 \mu \mathrm{m}$.

In conclusion we can state that the required but hitherto unattained power stability of 2 $\times 10^{-9} \mathrm{~Hz}^{-1 / 2}$ at $10 \mathrm{~Hz}$ for the Advanced LIGO has been achieved by scaling the number of photodiodes, reducing the beam jitter coupling, and by the use of low-noise electronics. We demonstrated an OOL relative power noise of $2.4 \times 10^{-9} \mathrm{~Hz}^{-1 / 2}$ (1.7 $\times 10^{-9} \mathrm{~Hz}^{-1 / 2}$ with subtracted OOL detector noise) at $10 \mathrm{~Hz}$, with no line harmonics present in the mea- surement. The power stability was prone to be limited by the beam jitter especially at mechanical resonance frequencies, but the coupling coefficient has been kept small by appropriate alignment.

The detector is ideal to be used in the upcoming generation of GW detectors owing to the demonstrated performance, the low back reflection/scatter design, and the vacuum compatibility. Since the limiting noise sources were identified, the power stability can probably be further improved in the future. At low frequencies the electronic noise needs to be lowered and at high frequencies the total detected photocurrent has to be increased to reduce the relative shot noise. Further, the techniques used in this Letter can be combined with advanced powerstabilization techniques such as the optical ac coupling [12] to reach even better power stabilities.

This research is supported by the Deutsche Forschungsgemeinschaft (DFG) and is part of Sonderforschungsbereich (SFB) No. 407 and Centre for Quantum Engineering and Space-Time Research (QUEST). The authors thank Albrecht Rüdiger for his helpful comments during the preparation of this Letter.

\section{References}

1. S. Rowan and J. Hough, Living Rev. Relativ. 3 (2000).

2. P. R. Saulson, Fundamentals of Interferometric Gravitational Wave Detectors (World Scientific, 1994).

3. K. Somiya, Y. Chen, S. Kawamura, and N. Mio, Phys. Rev. D 73, 122005 (2006).

4. S. Hild, H. Grote, J. Degallaix, S. Chelkowski, K. Danzmann, A. Freise, M. Hewitson, J. Hough, H. Lück, M. Prijatelj, K. A. Strain, J. R. Smith, and B. Willke, Class. Quantum Grav. 26, 055012 (2009).

5. P. Fritschel, Proc. SPIE 4856, 282 (2003).

6. F. Seifert, P. Kwee, M. Heurs, B. Willke, and K. Danzmann, Opt. Lett. 31, 2000 (2006).

7. B. W. Barr, K. A. Strain, and C. J. Killow, Class. Quantum Grav. 22, 4279 (2005).

8. J. Rollins, D. Ottaway, M. Zucker, R. Weiss, and R. Abbott, Opt. Lett. 29, 1876 (2004).

9. O. Jennrich, G. Newton, K. D. Skeldon, and J. Hough, Opt. Commun. 205, 405 (2002).

10. N. Uehara and K. Ueda, Opt. Lett. 19, 728 (1994).

11. B. Willke, N. Uehara, E. Gustafson, R. L. Byer, P. J. King, S. U. Seel, and R. L. Savage, Opt. Lett. 23, 1704 (1998).

12. P. Kwee, B. Willke, and K. Danzmann, Opt. Lett. 33, 1509 (2008). 